\title{
Combination therapy of brain radiotherapy and EGFR-TKIs is more effective than TKls alone for EGFR-mutant lung adenocarcinoma patients with asymptomatic brain metastasis
}

Yanxin Chen ${ }^{1,2}$, Jianping Wei ${ }^{1}$, Jing Cai ${ }^{1}$ and Anwen Liu ${ }^{1,2^{*}}$

\begin{abstract}
Background: The treatment strategy for brain metastasis (BM) in patients with epidermal growth factor receptor (EGFR) - mutant lung adenocarcinoma (LAC) remains controversial. In the present study, we compared the efficacy of brain radiotherapy (RT) in combination with tyrosine kinase inhibitors (TKIs) and TKIs alone for advanced LAC patients with EGFR mutations and BM.

Methods: We retrospectively studied 78 patients diagnosed with EGFR-mutant LAC who developed BM. These patients were divided into two groups: 49 patients in the combination treatment group who received brain RT in combination with EGFR-TKIs (including 23 patients with asymptomatic BM before RT); 29 patients in the TKI group who received EGFR-TKI targeted therapy alone (including 22 patients with asymptomatic BM before TKI treatment).

Results: The median intracranial progression-free survival (iPFS) of the combination treatment group was longer than that of the TKI alone group (21.5 vs. 15 months; $P=0.036)$. However, there were no significant differences in median progression-free survival (PFS, 12 vs. 13 months; $P=0.242$ ) and median overall survival (mOS, 36 vs. 23 months; $P=0.363$ ) between the two groups. Further analysis of asymptomatic BM showed that both the median iPFS and the mOS of the combination treatment group were significantly longer than for the TKI alone group (iPFS, 21.5 vs. 14.8 months, $P=0.026$; mOS, 36 vs. 23 months, $P=0.041$ ). Cox multivariate regression analysis found no independent adverse predictors of iPFS in all patients.

Conclusions: The synchronous combination of brain RT and TKIs was superior to EGFR-TKIs alone for EGFR-mutant LAC patients with BM. The combination treatment group exhibited longer PPFS, while the PFS and OS were not significantly different between the two groups. In addition, the combination treatment could result in better iPFS and OS in those with asymptomatic BM. Therefore, addition of brain RT was useful for intracranial metastatic lesions.
\end{abstract}

Keywords: EGFR-TKIs, Lung adenocarcinoma, Brain metastasis, Radiotherapy

\footnotetext{
* Correspondence: awliu666@163.com

'Department of oncology, The second affiliated hospital of Nanchang University, Jiangxi province, Nanchang 330006, China

${ }^{2}$ Jiangxi key laboratory of clinical translational cancer research, The second

affiliated hospital of Nanchang University, Jiangxi province, Nanchang

330006, China
}

(c) The Author(s). 2019 Open Access This article is distributed under the terms of the Creative Commons Attribution 4.0 International License (http://creativecommons.org/licenses/by/4.0/), which permits unrestricted use, distribution, and reproduction in any medium, provided you give appropriate credit to the original author(s) and the source, provide a link to the Creative Commons license, and indicate if changes were made. The Creative Commons Public Domain Dedication waiver (http://creativecommons.org/publicdomain/zero/1.0/) applies to the data made available in this article, unless otherwise stated. 


\section{Background}

An estimated 18.1 million new cases of cancer and 9.6 million cancer-related deaths occurred as of 2018 [1]. Lung cancer is the most commonly diagnosed cancer (11.6\%) and also the leading cause of cancer-related death (18.4\% of all cancer deaths) [1]. During the course of the disease, $22-54 \%$ of non-small-cell lung carcinoma (NSCLC) patients develop brain metastasis (BM) [2] [3]. Studies have shown that the incidence of BM in lung adenocarcinoma (LAC) is higher than that in other subtypes of NSCLC. About $45-52 \%$ of LAC patients develop BM during the course of the disease [4]. BM is a common complication in LAC patients and an important cause of morbidity and mortality [5]. In general, the prognosis of patients with $\mathrm{BM}$ still remains poor. The epidermal growth factor receptor (EGFR) gene plays a critical role in regulating normal cell proliferation, apoptosis, and other cellular roles $[6,7]$. Studies have shown that EGFR mutation is significantly associated with the risk of $\mathrm{BM}$ after initial diagnosis and radical resection of LAC [8]. Patients with EGFR mutations are more vulnerable to BM than those with wild-type EGFR. At initial diagnosis [9], BM is found in approximately $25 \%$ of patients with EGFR mutations. Therefore, it is urgently necessary to develop reasonable and effective treatments to address this.

The development of radiotherapy (RT) and targeted therapy, and particularly, the combination of RT and targeted therapy, in recent years, has greatly prolonged the median overall survival (OS) and median progressionfree survival (PFS) for NSCLC patients with BM [10]. For EGFR-mutant NSCLC patients with BM, tyrosine kinase inhibitors (TKIs) can effectively control intracranial position of the disease [11]. Brain RT can also effectively control intracranial lesions [12]. Based on the advantages of these individual treatments, we posited that a combination therapy might be effective. However, based on currently available data, the efficacy of such a combination remains controversial. Some studies have shown that brain RT in combination with EGFR-TKIs is more effective than TKIs alone [13]. However, other studies have shown that TKIs in combination with RT has no beneficial effects on intracranial PFS (iPFS) or OS [14].

Furthermore, patients with BM but no intracranial symptoms do not require immediate relief, and suitable treatment options are still disputed. EGFR-TKIs have been used for the treatment of asymptomatic BM. However, only a few studies have assessed the effects of EGFR-TKIs in combination with RT. In the present study, we aimed to explore whether combination therapy of TKIs and RT could benefit asymptomatic BM.

We retrospectively evaluated the efficacy of combination therapy and TKIs alone in the treatment of LAC patients with BM and EGFR mutations. We also evaluated the efficacy of these two therapeutic regimens in asymptomatic BM.

\section{Methods \\ Patients}

A total of 391 patients were diagnosed with LAC between April 2014 and June 2018 at the Second Affiliated Hospital of Nanchang University, of which 78 patients were diagnosed with stage IV LAC, and these patients were also detected with EGFR mutation and BM. These 78 patients with $\mathrm{BM}$ at preliminary diagnosis were retrospectively enrolled and analyzed in the present study. The inclusion criteria were set as follows: 1) LAC diagnosis by percutaneous lung biopsy or fiberoptic bronchoscopy; or reconfirmation of a pathological section as LAC after consultation in our hospital, followed by EGFR mutation diagnosis by genetic test; 2) older than 18 years old; 3 ) BM diagnosis by craniocerebral magnetic resonance imaging (MRI); 4) type of comparison: TKIs alone or combination of brain RT and TKIs. The exclusion criteria were set as follows: 1) patients who developed BM after taking EGFR-TKIs; 2) patients who did not receive EGFR-TKIs after stereotactic radiosurgery (SRS) or whole brain radiotherapy (WBRT); and 3) patients who received TKIs before or after brain RT.

Clinical information of patients was collected, including age, gender, smoking status, EGFR mutation status, number of BM, extracranial metastasis, EGFR-TKI drugs, type of brain RT, an update of the Graded Prognostic Assessment for Lung Cancer using Molecular Markers (lung-molGPA), Karnofsky Performance Status (KPS) score, and the location of the primary disease. Importantly, the absence or presence of intracranial symptoms here refers to the beginning of treatment, rather than the entire course of disease progression. Asymptomatic BM was defined as no increased intracranial pressure, dizziness, headache, nausea or vomiting, visual impairment, mental symptoms, and seizures or signs of focal neurological symptoms, regardless of whether there are symptomatic in other parts, including the lungs. Age, number of BM, extracranial metastasis, and lung-mol GPA scores reflected the current status of all patients who received treatment. The type of EGFR mutation was divided into the common EGFR mutations: exon 19 deletion (19del) and Leu858Arg point mutation (L858R). Rare EGFR mutations were defined as those other than 19del and L858R. Primary intracranial disease progression means that other systemic lesions were stable, while intracranial lesions progressed. A total of 78 patients were treated with EGFR-TKIs (gefitinib $250 \mathrm{mg}$ qd; erlotinib $150 \mathrm{mg}$ qd; icotinib 125 $\mathrm{mg}$, tid). For the brain radiation group, the Elekta Versa HD medical linear accelerator and the Monaco planning 
system were used. The total radiation dose for WBRT was $30 \mathrm{~Gy}$ administered in 10 fractions (once a day, 5 days per week, 3 Gy each time). The total dose for SRS was $25 \mathrm{~Gy}$ administered in 5 fractions (once a day, 5 days per week, $5 \mathrm{~Gy}$ each time), $30 \mathrm{~Gy}$ administered in 5 fractions (once a day, 5 days per week, 6 Gy each time), or 35 Gy administered in 5 fractions (once a day, 5 days per week, 7 Gy each time). Each patient underwent laboratory and imaging examinations, including CT scans of the chest and upper abdomen, computed tomography (ECT) of the bone, and MRI of the brain. Patients were evaluated for efficacy 1 month after the end of treatment, followed by 2 months and then every 3 months. The therapeutic effect was evaluated by brain MRI, chest CT and upper abdominal CT. Tumor response was assessed by the Response Evaluation Criteria in Solid Tumors (RECIST) 1.1.

\section{Statistical analysis}

The iPFS was defined as the time from the initiation of RT in combination with EGFR-TKIs or TKIs alone to the time of intracranial progression or death without documented progression, the last follow-up time for patients who did not progress or died was a censored value. PFS was defined as the time from the onset of treatment to any disease progression in the body or death without documented progression, the last followup time for patients who did not progress or died was a censored value. OS was defined as the time from the initiation of RT in combination with EGFR-TKIs or TKIs alone to death or last follow-up if they were still alive. Survival analysis was performed using Kaplan-Meier curves. The effects of potential variables on PFS were assessed by univariate analysis. Multivariate testing was performed by Cox regression analysis. Statistical analysis was performed by using SPSS software version 22.0.

\section{Results}

\section{Patients' characteristics}

We included 613 patients who were diagnosed with LAC from April 2014 to June 2018 at the Second Affiliated Hospital of Nanchang University. Among them, 391 LAC patients were selected according to the inclusion criteria. Finally, 78 LAC patients diagnosed with EGFR mutations who developed BM were enrolled in the present study (Fig. 1). Table 1 shows the baseline characteristics of patients. Among them, 49 (62.8\%) received a combination therapy of brain RT and EGFR-TKIs, and the other 29 (37.1\%) received EGFR-TKI targeted therapy alone. Our data showed that 45 patients $(57.7 \%)$ had asymptomatic $\mathrm{BM}$ at the beginning of treatment, of which, 22 patients were treated with TKIs alone and 23 patients received the combination therapy of TKIs and RT. Table 2 shows the baseline characteristics of these patients.

The final follow-up date of the study was October 29, 2018. At the time of last follow-up, 28 patients survived without signs of disease progression, 21 patients exhibited

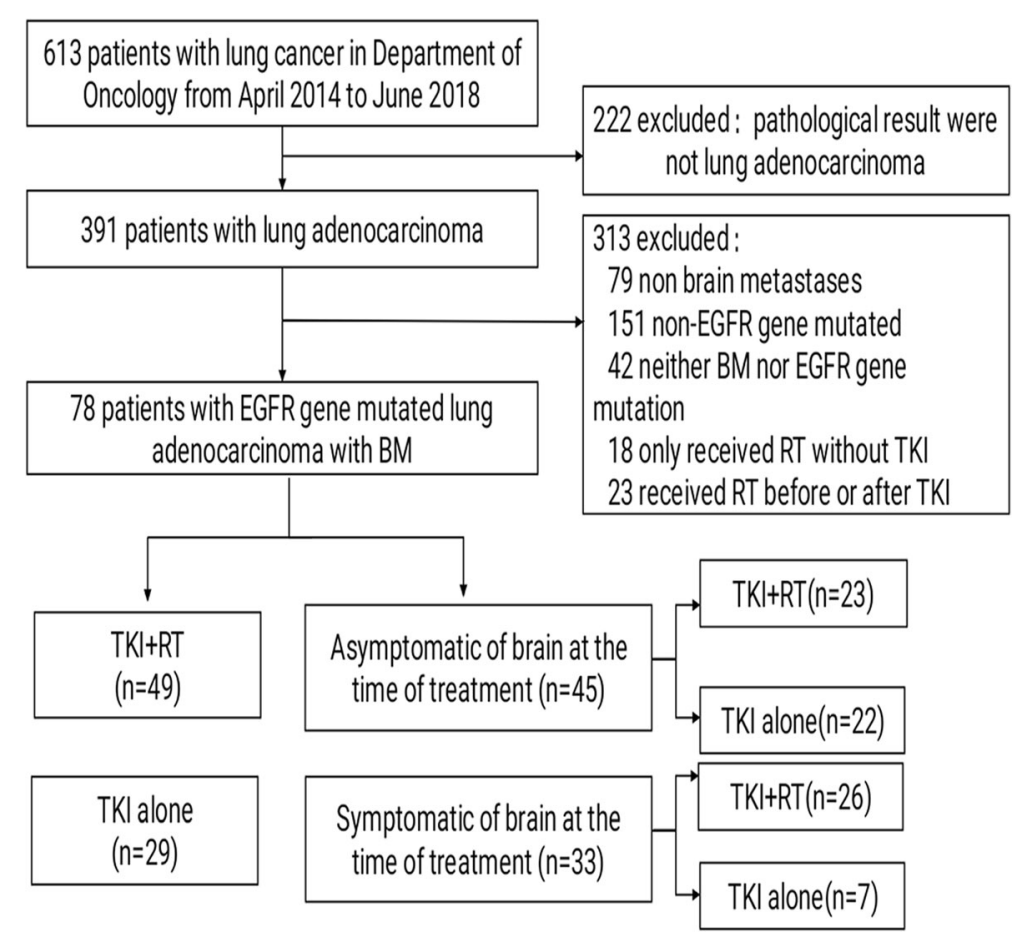

Fig. 1 The flow chart of the patient queue 
Table 1 Clinical and Molecular Characteristics of Included Patients

\begin{tabular}{|c|c|c|c|c|c|}
\hline \multirow[b]{2}{*}{ Characteristic } & \multicolumn{2}{|c|}{ TKI alone } & \multicolumn{2}{|l|}{$T K I+R T$} & \multirow[b]{2}{*}{$p$ Value } \\
\hline & $(n=29)$ & $\%$ & $(n=49)$ & $\%$ & \\
\hline Age (years) & & & & & 0.639 \\
\hline Median & 59 & & 59 & & \\
\hline Range & $32-74$ & & $35-83$ & & \\
\hline$<65$ & 21 & 72.4 & 33 & 67.3 & \\
\hline$\geq 65$ & 8 & 27.6 & 16 & 32.7 & \\
\hline Gender & & & & & 0.729 \\
\hline Male & 13 & 44.8 & 20 & 40.8 & \\
\hline Female & 16 & 55.2 & 29 & 59.2 & \\
\hline Smoking history & & & & & 0.729 \\
\hline Never or light & 16 & 55.2 & 29 & 59.2 & \\
\hline Heavy & 13 & 44.8 & 20 & 40.8 & \\
\hline EGFR mutation & & & & & 0.323 \\
\hline Del19 & 9 & 31.0 & 22 & 44.9 & \\
\hline L858r & 18 & 62.0 & 26 & 53.1 & \\
\hline Other & 2 & 7.0 & 1 & 2.0 & \\
\hline BM no. at time of diagnosis & & & & & 0.292 \\
\hline$\leq 3$ & 16 & 55.2 & 21 & 42.9 & \\
\hline$>3$ & 13 & 44.8 & 28 & 57.1 & \\
\hline Extracranial metastases & & & & & 0.454 \\
\hline Yes & 24 & 82.8 & 37 & 75.5 & \\
\hline No & 5 & 17.2 & 12 & 24.5 & \\
\hline Intracranial Symptoms & & & & & 0.012 \\
\hline Without & 22 & 75.9 & 23 & 46.9 & \\
\hline With & 7 & 24.1 & 26 & 53.1 & \\
\hline Lung-mol GPA classification & & & & & 0.339 \\
\hline $0-1$ & 1 & 3.5 & 2 & 4.1 & \\
\hline $1.5-2$ & 9 & 31.0 & 12 & 24.5 & \\
\hline $2.5-3$ & 12 & 41.4 & 22 & 44.9 & \\
\hline $3.5-4$ & 7 & 24.1 & 13 & 26.5 & \\
\hline Primary tumor location & & & & & 0.128 \\
\hline Left Lung & 17 & 58.6 & 20 & 40.8 & \\
\hline Right Lung & 12 & 41.4 & 29 & 59.2 & \\
\hline KPS score(\%) & & & & & 0.801 \\
\hline$<80$ & 6 & 20.7 & 9 & 18.4 & \\
\hline$\geq 80$ & 23 & 79.3 & 40 & 81.6 & \\
\hline
\end{tabular}

signs of disease progression, 27 patients had died of disease progression, one patient had died of unrelated causes, and one patient was lost during follow-up.

In the present study, 49 of 78 patients received combination therapy of RT and TKIs (WBRT in 35 and SBRT in 14), and 29 patients received TKIs alone. Intracranial progression was detected in 31 of the 78 patients (39.7\%). Intracranial progression occurred in $41.8 \%$ (12
Table 2 Clinical and Molecular Characteristics of patients with asymptomatic brain metastases

\begin{tabular}{|c|c|c|c|c|c|}
\hline & $T K I+R T$ & & TKI alone & & \\
\hline Characteristic & $(n=23)$ & $\%$ & $(n=22)$ & $\%$ & $p$ Value \\
\hline Age (years) & & & & & 0.608 \\
\hline Median & 61 & & 59 & & \\
\hline Range & $44-75$ & & $41-74$ & & \\
\hline$<65$ & 14 & 60.9 & 15 & 68.2 & \\
\hline$\geq 65$ & 9 & 39.1 & 7 & 31.8 & \\
\hline Gender & & & & & 0.295 \\
\hline Male & 10 & 43.5 & 13 & 59.1 & \\
\hline Female & 13 & 56.5 & 9 & 40.9 & \\
\hline Smoking history & & & & & 0.295 \\
\hline Never or light & 13 & 56.5 & 9 & 40.9 & \\
\hline Heavy & 10 & 43.5 & 13 & 59.1 & \\
\hline EGFR mutation & & & & & 0.155 \\
\hline Del19 & 12 & 52.2 & 16 & 72.7 & \\
\hline L858r & 11 & 47.8 & 6 & 27.3 & \\
\hline Other & 0 & 0 & 0 & 0 & \\
\hline BM No. at time of diagnosis & & & & & 0.463 \\
\hline$\leq 3$ & 14 & 60.9 & 11 & 50.0 & \\
\hline$>3$ & 9 & 39.1 & 11 & 50.0 & \\
\hline Extracranial metastases & & & & & 0.477 \\
\hline Yes & 18 & 78.3 & 19 & 86.4 & \\
\hline No & 5 & 21.7 & 3 & 13.6 & \\
\hline Lung-mol GPA classification & & & & & 0.266 \\
\hline $0-1$ & 0 & 0 & 0 & 0 & \\
\hline $1.5-2$ & 3 & 13.1 & 6 & 27.3 & \\
\hline $2.5-3$ & 13 & 56.5 & 10 & 45.4 & \\
\hline $3.5-4$ & 7 & 30.4 & 6 & 27.3 & \\
\hline
\end{tabular}

out of 29) of the patients who received EGFR-TKIs alone, compared with $38.7 \%$ (19 out of 49 ) for patients who received combination therapy of EGFR-TKIs and brain RT. Primary intracranial disease progression was noted in $27.6 \%$ (8 out of 29) of patients who received TKIs alone, compared to $18.3 \%$ (9 out of 49 ) in patients receiving combination therapy.

\section{Survival outcomes}

The median PFS of the study population was 11 months. The median iPFS of patients receiving RT + TKIs was 21.5 months, which was significantly longer than that of those receiving EGFR-TKIs alone (median iPFS, 15 months, $P=0.036)$. However, the median PFS (mPFS, 12 months versus 13 months; $P=0.242$ ) and mOS (36 months versus 23 months, $P=0.363$ ) were not significant different in these two groups, although the 
$\mathrm{mPFS}$ and $\mathrm{mOS}$ in the combination treatment group were higher (Fig. 2).

For patients with asymptomatic BM, the median iPFS was 21.5 months for patients who received RT + TKIs $(n=23)$ and 14.8 months for patients who received EGFR-TKIs alone $(n=22, P=0.026)$. The OS was prolonged in patients who received RT + TKIs (36 months, $P=0.041)$ compared with those who received TKIs alone (23 months) (Fig. 3).

Cox multivariate regression analysis found no independent adverse predictors of iPFS in all patients (Table 3).

\section{Discussion}

Based on our small-scale retrospective study, we could conclude the following: 1) a combination therapy of RT and TKIs could improve iPFS, while OS and PFS were not significantly prolonged compared with TKIs alone; 2) for patients with asymptomatic BM, the iPFS and OS of the combination therapy group were longer compared with the TKIs alone group.

Multiple retrospective studies have reported similar results [13, 15-17]. For example, a systematic review and meta-analysis consisting of 12 studies found that in EGFR-mutant NSCLC patients who develop BM, cranial RT followed by TKIs improved iPFS compared with upfront TKI, showing that the use of upfront EGFRTKIs and delay of RT were associated with poor PFS [17]. Several mechanisms can explain the combined effect of TKI + WBRT on BM of NSCLC patients with EGFR mutation. Firstly, EGFR-TKI can inhibit the proliferation of tumor cells, inhibit the apoptosis pathway, and suppress DNA repair capability, making tumor cells more sensitive to RT $[18,19]$. Secondly, RT can increase the effective concentration of TKIs by enhancing the blood brain barrier (BBB) permeability [20]. Finally, radiation can reduce the probability of the $\mathrm{T} 790 \mathrm{M} \mathrm{mu}$ tation $[21,22]$.

Magnuson et al. conducted a multi-institutional analysis consisting of 351 EGFR-mutant NSCLC patients who developed BM. The patients were divided into three groups: SRS followed by EGFR-TKI, WBRT followed by EGFR-TKI, or EGFR-TKI followed by SRS or WBRT. This analysis, however, demonstrated that the iPFS of these three groups was similar at 23 months, 24 months, and 17 months, respectively [16]. Byeon et al. have also shown that there are no differences between these treatments [23], although, their study used sequential cranial $\mathrm{RT}$, in contrast to a synchronous combination of brain RT and TKIs in our study. Another study showed that based on the radiosensitizing effect, the duration of opening the $\mathrm{BBB}$, and the reproductive death, it is reasonable to administer TKIs either concurrently or one week before RT [24]. Moreover, in a study by Yang et al., with 85 patients in the icotinib group and 91 patients in the WBRT group, the median iPFS of NSCLC patients with EGFR mutation and BM were 4.8 months and 10.0 months $(P<0.05)$, while the median OS were 20.5 months and 18.0 months $(P>0.05)$. Therefore, TKIs alone may be insufficient to treat BM of NSCLC [25].

Treatment strategies remain uncertain for patients with asymptomatic BM. In a study by Chen et al., combination RT showed no significant changes in intracranial TTP $(P=0.193)$ for asymptomatic patients [26]. Liu et al. reported that first-line treatment using brain RT fails to lengthen the survival time of patients with EGFR mutation and asymptomatic BM [27]. Based on the high intracranial response rates, TKIs alone have been proposed as initial treatment in patients with EGFR mutations and asymptomatic BM [28]. However, this approach can be associated with a higher risk of subsequent intracranial relapse. The use of primary TKIs can ameliorate the adverse effects of RT; however, it is
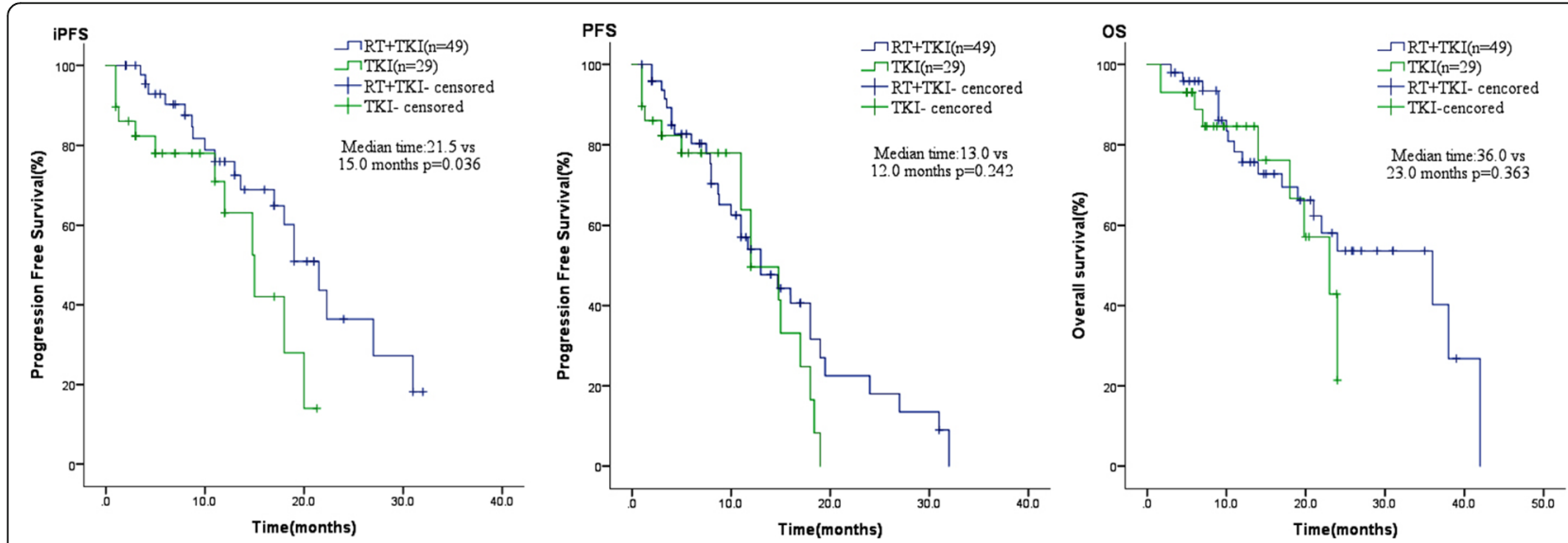

Fig. 2 Combination therapy group had similar PFS and OS, but better iPFS than only TKIs therapy group in LAC patients with EGFR-mutant and BM 


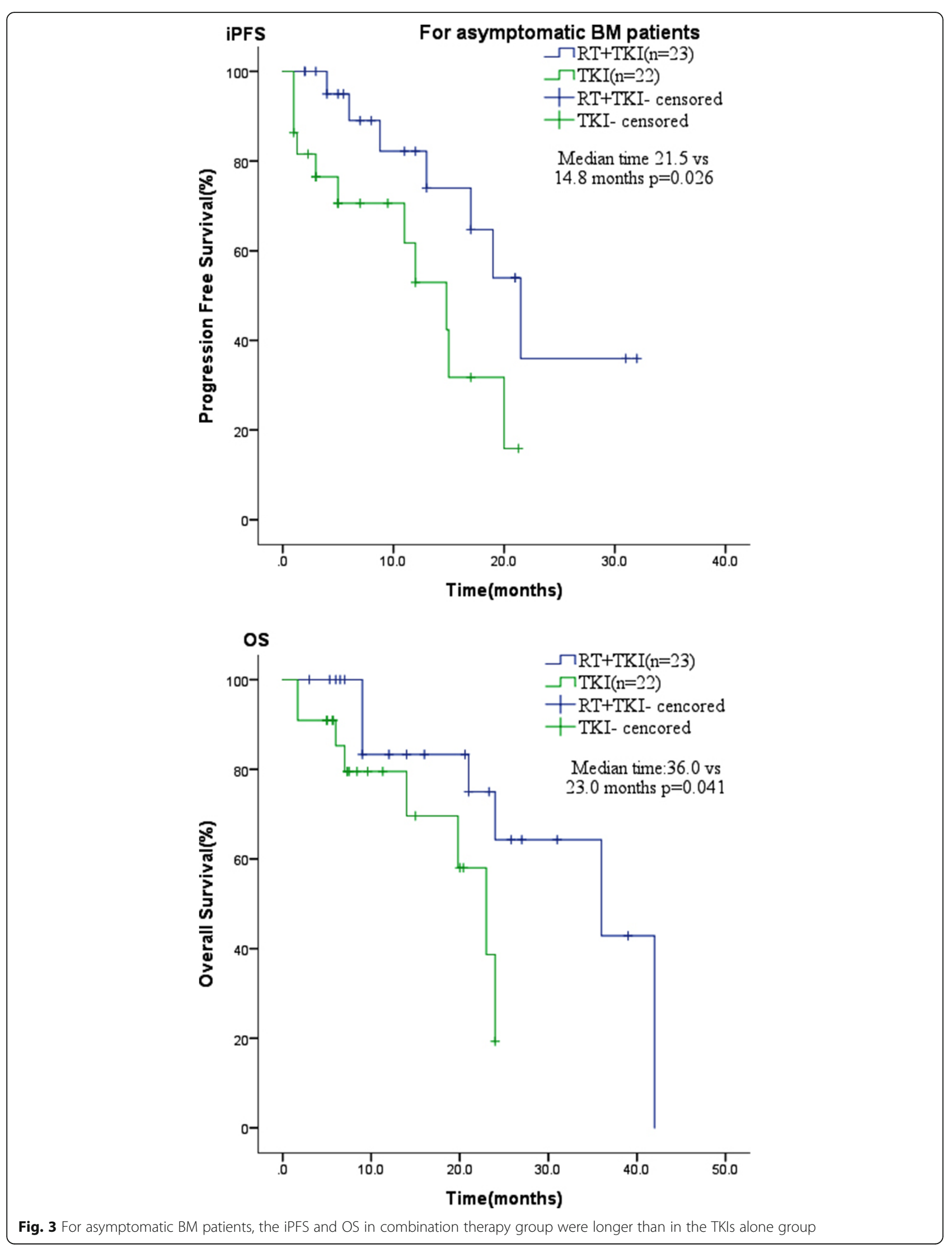


Table 3 Multivariate analysis of prognostic factors for iPFS in 78 patients

\begin{tabular}{|c|c|c|c|c|}
\hline & \multirow[t]{2}{*}{$P$} & \multirow[t]{2}{*}{$\mathrm{HR}$} & \multicolumn{2}{|c|}{$95.0 \% \mathrm{Cl}$ for $\mathrm{HR}$} \\
\hline & & & Lower & Upper \\
\hline Gender (male vs. female) & 0.137 & 0.16 & 0.02 & 1.78 \\
\hline Age (<65y vs. $\geq 65 y)$ & 0.890 & 0.94 & 0.37 & 2.34 \\
\hline Smoking history (never vs. smoking) & 0.134 & 5.57 & 0.59 & 52.53 \\
\hline EGFR mutation (L858r vs. Del19 vs. rare mutations) & 0.665 & 0.83 & 0.36 & 1.91 \\
\hline BM No. ( $\leq 3$ vs. $>3)$ & 0.315 & 0.63 & 0.26 & 1.55 \\
\hline Metastases (B vs. B + E) & 0.740 & 0.82 & 0.26 & 2.58 \\
\hline Intracranial symptom (have vs. No) & 0.267 & 0.60 & 0.24 & 1.48 \\
\hline First-line treatment (Yes vs. No) & 0.445 & 1.55 & 0.51 & 4.74 \\
\hline KPS score $(<80 \%$ vs $\geq 80 \%)$ & 0.918 & 0.94 & 0.26 & 3.37 \\
\hline
\end{tabular}

No. Number, $B$ brain, $B+E$ brain and extracranial metastasis

unlikely to they can completely abrogate the need for subsequent RT. In addition, asymptomatic patients may have lower tumor load, stronger physical condition, and less systemic metastasis. Therefore, effective control of intracranial lesions is more meaningful for long-term survival of patients. For EGFR-mutant LAC patients with BM, cranial RT in combination with TKIs is a possible strategy, that may improve PFS and OS compared with TKIs alone. Wang et al. have also reported similar results, that delayed brain RT may lead to lower iPFS in NSCLC patients with EGFR mutation and asymptomatic BM (0.032) [29].

The TKIs used in our study included gefitinib, erlotinib, and icotinib. However, osimertinib has demonstrated a greater penetrating capacity of the mouse BBB compared with gefitinib, rociletinib, and afatinib, and could achieve sustained tumor regression in an EGFR mutated PC9 mouse model of BM [30]. Studies also found that osimertinib combined with RT could significantly reduce the proliferation of NSCLC cells harboring T790 M/L858R mutations in vitro and in vivo, reduce cell cycle arrest in G2/M phase, and could block RT-induced DNA double-strand breaks (DSB) repair, demonstrating its role in radiosensitivity [31]. A double-blind, phase III trial found that the frequency of central nervous system progression was lower in the osimertinib group compared with the standard EGFR-TKI group [32]. Two randomized phase II trials of Osimertinib with or without SRS for the treatment of EGFR mutant NSCLC with BM (NCT03497767 and NCT03769103) are about to begin, and we are looking forward to their results. In conclusion, osimertinib in combination with cranial RT may have a greater benefit in LAC patients with BM and EGFR mutation, and further studies are needed to assess its efficacy.

Our current study has certain limitations: (i) we only included patients from a single institution, and the patient population was, thus, relatively small; (ii) due to the retrospective nature of our study, undefined biases and/or confounding factors may have influenced clinical outcomes.

\section{Conclusions}

Collectively, compared with EGFR-TKI treatment alone, combination therapy of TKIs and RT could significantly prolong iPFS. For patients with asymptomatic BM, the combination therapy showed beneficial effects on iPFS and OS, highlighting the usefulness of RT. Although combination therapy has grown in popularity in recent years, more prospective studies are needed to analyze different populations in order to achieve effective treatment.

\section{Abbreviations}

BM: Brain metastases; EGFR: Epidermal growth factor receptor; KPS: Karnofsky performance status; LAC: Lung adenocarcinoma; lung-molGPA: An update of the graded prognostic assessment for lung cancer using molecular markers; NSCLC: Non-small cell lung cancers; OS: Overall survival; PFS: Progression free survival; RT: Radiotherapy;i SRS: Stereotactic radiosurgery; TKI: Tyrosine kinase inhibitors; WBRT: Whole brain radiotherapy

\section{Acknowledgments}

We kindly thank LH for providing useful comments, participating in revision of the manuscript, and reediting the resubmitted manuscript for grammar and wording. We also kindly thank the editor and reviewers for their careful review. and valuable comments, which have significantly improved the manuscript.

\section{Authors' contributions}

YXC participated in the case collection, drafting, and wrote the manuscript: JPW made useful comments and participated in revising the manuscript; JC designed the study and performed the statistical analysis; AWL participated in the analysis and interpretation of the data, as well as in drafting and revising all versions of the manuscript. All authors have read and approved the final version for publication.

\section{Funding}

No funding was obtained for this report.

\section{Availability of data and materials}

The datasets used and/or analyzed during the current study are available from the corresponding author on reasonable request.

\section{Ethics approval and consent to participate}

The study was approved by the Ethics Committee of The Second Affiliated Hospital of Nanchang University. Written informed consents were obtained 
from all patients to perform radiotherapy or targeted therapy. Given that this is a retrospective study, we did not obtain written informed consents from all patients to participate in this study.

\section{Consent for publication}

Not applicable.

\section{Competing interests}

The authors declare that they have no competing interests.

Received: 18 January 2019 Accepted: 31 July 2019

Published online: 09 August 2019

\section{References}

1. Bray F, Ferlay J, Soerjomataram I, Siegel RL, Torre LA, Jemal A. Global cancer statistics 2018: GLOBOCAN estimates of incidence and mortality worldwide for 36 cancers in 185 countries. CA Cancer J Clin. 2018;68(6):394-424.

2. Barnholtz-Sloan JS, Sloan AE, Davis FG, Vigneau FD, Lai P, Sawaya RE. Incidence proportions of brain metastases in patients diagnosed (1973 to 2001) in the metropolitan Detroit Cancer surveillance system. J Clin Oncol. 2004;22(14):2865-72

3. Lombardi G, Di Stefano AL, Farina P, Zagonel V, Tabouret E. Systemic treatments for brain metastases from breast cancer, non-small cell lung cancer, melanoma and renal cell carcinoma: an overview of the literature. Cancer Treat Rev. 2014;40(8):951-9.

4. Jemal A, Bray F, Center MM, Ferlay J, Ward E, Forman D. Global cancer statistics. CA Cancer J Clin. 2011;61(2):69-90.

5. Sul J, Posner JB. Brain metastases: epidemiology and pathophysiology. Cancer Treat Res. 2007;136:1-21.

6. Lynch TJ, Bell DW, Sordella R, Gurubhagavatula S, Okimoto RA, Brannigan BW, Harris PL, Haserlat SM, Supko JG, Haluska FG, et al. Activating mutations in the epidermal growth factor receptor underlying responsiveness of nonsmall-cell lung cancer to gefitinib. N Engl J Med. 2004;350(21):2129-39.

7. Paez JG, Janne PA, Lee JC, Tracy S, Greulich H, Gabriel S, Herman P, Kaye FJ, Lindeman $\mathrm{N}$, Boggon TJ, et al. EGFR mutations in lung cancer: correlation with clinical response to gefitinib therapy. Science (New York, NY). 2004; 304(5676):1497-500.

8. Shin DY, Na II, Kim CH, Park S, Baek H, Yang SH. EGFR mutation and brain metastasis in pulmonary adenocarcinomas. J Thorac Oncol. 2014;9(2):195-9.

9. Rangachari D, Yamaguchi N, VanderLaan PA, Folch E, Mahadevan A, Floyd SR, Uhlmann EJ, Wong ET, Dahlberg SE, Huberman MS, et al. Brain metastases in patients with EGFR-mutated or ALK-rearranged non-small-cell lung cancers. Lung Cancer (Amsterdam, Netherlands). 2015;88(1):108-11.

10. Zheng H, Liu QX, Hou B, Zhou D, Li JM, Lu X, Wu QP, Dai JG. Clinical outcomes of WBRT plus EGFR-TKIs versus WBRT or TKIs alone for the treatment of cerebral metastatic NSCLC patients: a meta-analysis. Oncotarget. 2017:8(34):57356-64.

11. Mok TS, Wu YL, Thongprasert S, Yang CH, Chu DT, Saijo N, Sunpaweravong P, Han B, Margono B, Ichinose Y, et al. Gefitinib or carboplatin-paclitaxel in pulmonary adenocarcinoma. N Engl J Med. 2009;361(10):947-57.

12. Scoccianti S, Ricardi U. Treatment of brain metastases: review of phase III randomized controlled trials. Radiother Oncol. 2012;102(2):168-79.

13. Zeng YD, Zhang L, Liao $H$, Liang $Y, X u F$, Liu JL, Dinglin XX, Chen LK. Gefitinib alone or with concomitant whole brain radiotherapy for patients with brain metastasis from non-small-cell lung cancer: a retrospective study. Asian Pac J Cancer Prev. 2012;13(3):909-14.

14. Jiang T, Su C, Li X, Zhao C, Zhou F, Ren S, Zhou C, Zhang J. EGFR TKls plus WBRT demonstrated no survival benefit other than that of TKIs alone in patients with NSCLC and EGFR mutation and brain metastases. J Thorac Oncol. 2016;11(10):1718-28.

15. Zhu Q, Sun Y, Cui Y, Ye K, Yang C, Yang D, Ma J, Liu X, Yu J, Ge H. Clinical outcome of tyrosine kinase inhibitors alone or combined with radiotherapy for brain metastases from epidermal growth factor receptor (EGFR) mutant non small cell lung cancer (NSCLC). Oncotarget. 2017;8(8):13304-11.

16. Magnuson WJ, Lester-Coll NH, Wu AJ, Yang TJ, Lockney NA, Gerber NK, Beal K, Amini A, Patil T, Kavanagh BD, et al. Management of Brain Metastases in tyrosine kinase inhibitor-naive epidermal growth factor receptor-mutant non-small-cell lung Cancer: a retrospective multi-institutional analysis. J Clin Oncol. 2017;35(10):1070-7.

17. Soon YY, Leong CN, Koh WY, Tham IW. EGFR tyrosine kinase inhibitors versus cranial radiation therapy for EGFR mutant non-small cell lung cancer with brain metastases: a systematic review and meta-analysis. J Clin Oncol. 2015;114(2):167-72.

18. Tanaka T, Munshi A, Brooks C, Liu J, Hobbs ML, Meyn RE. Gefitinib radiosensitizes non-small cell lung cancer cells by suppressing cellular DNA repair capacity. Clin Cancer Res. 2008;14(4):1266-73.

19. Chinnaiyan P, Huang S, Vallabhaneni G, Armstrong E, Varambally S, Tomlins SA, Chinnaiyan AM, Harari PM. Mechanisms of enhanced radiation response following epidermal growth factor receptor signaling inhibition by erlotinib (Tarceva). Cancer Res. 2005;65(8):3328-35.

20. d'Avella D, Cicciarello R, Angileri FF, Lucerna S, La Torre D, Tomasello F. Radiation-induced blood-brain barrier changes: pathophysiological mechanisms and clinical implications. Acta Neurochir Suppl. 1998;71:282-4.

21. Li J, Wu X, Wang Z, Shen Z, Sun N, Zhu X. lonizing Radiation Reduces TKI Resistance Caused by T790M Mutation in NSCLC Cell Lines. Zhongguo Fei Ai Za Zhi. 2015;18(8):475-80.

22. Ahsan A. Mechanisms of resistance to EGFR tyrosine kinase inhibitors and therapeutic approaches: an update. Adv Exp Med Biol. 2016;893:137-53.

23. Byeon S, Ham JS, Sun JM, Lee SH, Ahn JS, Park K, Ahn MJ. Analysis of the benefit of sequential cranial radiotherapy in patients with EGFR mutant non-small cell lung cancer and brain metastasis. Med Oncol (Northwood, London, England). 2016;33(8):97.

24. Zhuang $\mathrm{H}$, Wang J, Zhao L, Yuan Z, Wang P. The theoretical foundation and research progress for WBRT combined with erlotinib for the treatment of multiple brain metastases in patients with lung adenocarcinoma. Int Cancer. 2013;133(10):2277-83.

25. Yang JJ, Zhou C, Huang Y, Feng J, Lu S, Song Y, Huang C, Wu G, Zhang L, Cheng $Y$, et al. Icotinib versus whole-BRAIN irradiation in patients with EGFR-mutant non-small-cell lung cancer and multiple BRAIN metastases (BRAIN): a multicentre, phase 3, open-label, parallel, randomised controlled trial. Lancet Respir Med. 2017;5(9):707-16.

26. Chen Y, Yang J, Li X, Hao D, Wu X, Yang Y, He C, Wang W, Wang J. First-line epidermal growth factor receptor (EGFR)-tyrosine kinase inhibitor alone or with whole-brain radiotherapy for brain metastases in patients with EGFRmutated lung adenocarcinoma. Cancer Sci. 2016;107(12):1800-5.

27. Liu S, Qiu B, Chen L, Wang F, Liang Y, Cai P, Zhang L, Chen Z, Liu S, Liu M, et al. Radiotherapy for asymptomatic brain metastasis in epidermal growth factor receptor mutant non-small cell lung cancer without prior tyrosine kinase inhibitors treatment: a retrospective clinical study. Radiat Oncol (London, England). 2015;10:118.

28. Jamal-Hanjani M, Spicer J. Epidermal growth factor receptor tyrosine kinase inhibitors in the treatment of epidermal growth factor receptor-mutant non-small cell lung cancer metastatic to the brain. Clin Cancer Res. 2012; 18(4):938-44.

29. Wang W, Song Z, Zhang Y. Efficacy of brain radiotherapy plus EGFR-TKI for EGFR-mutated non-small cell lung cancer patients who develop brain metastasis. Arch Med Sci. 2018;14(6):1298-307.

30. Ballard P, Yates JW, Yang Z, Kim DW, Yang JC, Cantarini M, Pickup K, Jordan A, Hickey M, Grist M, et al. Preclinical comparison of Osimertinib with other EGFR-TKIs in EGFR-mutant NSCLC brain metastases models, and early evidence of clinical brain metastases activity. Clin Cancer Res. 2016;22(20): 5130-40.

31. Wang N, Wang L, Meng X, Wang J, Zhu L, Liu C, Li S, Zheng L, Yang Z, Xing $L$, et al. Osimertinib (AZD9291) increases radiosensitivity in EGFR T790M nonsmall cell lung cancer. Oncol Rep. 2019;41(1):77-86.

32. Gregorc $V$, Lazzari $C$, Karachaliou N, Rosell R, Santarpia M: Osimertinib in untreated epidermal growth factor receptor (EGFR)-mutated advanced nonsmall cell lung cancer. Translat Lung Cancer Res 2018, 7(Suppl 2):S165-s170.

\section{Publisher's Note}

Springer Nature remains neutral with regard to jurisdictional claims in published maps and institutional affiliations. 